\title{
Research
}

Patricia Moreno-Peral, Sonia Conejo-Cerón, Juan de Dios Luna, Michael King, Irwin Nazareth, Carlos Martín-Pérez, Carmen Fernández-Alonso, María Isabel Ballesta-Rodríguez, Anna Fernández, José María Aiarzaguena, Carmen Montón-Franco, Juan Ángel Bellón and the predictD group

\section{Use of a personalised depression intervention in primary care to prevent anxiety:}

\author{
a secondary study of a cluster randomised trial
}

\begin{abstract}
Background

In the predictD-intervention, GPs used a personalised biopsychosocial programme to prevent depression. This reduced the incidence of major depression by $21.0 \%$, although the results were not statistically significant.
\end{abstract}

\section{Aim}

To determine whether the predictD-intervention is effective at preventing anxiety in primary care patients without depression or anxiety.

\section{Design and setting}

Secondary study of a cluster randomised trial with practices randomly assigned to either the predictD-intervention or usual care. This study was conducted in seven Spanish cities from October 2010 to July 2012

\section{Method}

In each city, 10 practices and two GPs per practice, as well as four to six patients every recruiting day, were randomly selected until there were 26-27 eligible patients for each GP. The endpoint was cumulative incidence of anxiety as measured by the PRIME-MD screening tool over 18 months.

\section{Results}

A total of 3326 patients without depression and 140 GPs from 70 practices consented and were eligible to participate; 328 of these patients were removed because they had an anxiety syndrome at baseline. Of the 2998 valid patients, 2597

$(86.6 \%)$ were evaluated at the end of the study. At 18 months, $10.4 \%$ (95\% Cl= $8.7 \%$ to $12.1 \%$ ) of the patients in the predictD-intervention group developed anxiety compared with $13.1 \%$ (95\% $\mathrm{Cl}=11.4 \%$ to $14.8 \%$ ) in the usual-care group labsolute difference $=-2.7 \%[95 \% \mathrm{Cl}=-5.1 \%$ to $-0.3 \%$ ]; $P=0.029$ ).

\section{Conclusion}

A personalised intervention delivered by GPs for the prevention of depression provided a modest but statistically significant reduction in the incidence of anxiety.

\section{Keywords}

anxiety; controlled clinical trial; depression;

primary care; primary prevention.

\section{INTRODUCTION}

The average 12-month prevalence of anxiety disorders is $6.7 \%$ in the general population, ${ }^{1}$ reaching $18.5 \%$ in patients in primary care. ${ }^{2}$ Between 2007 and 2017 . the burden of disease in terms of years lived with disability attributable to anxiety disorders increased by $12.4 \%$ and $13.6 \%$ for females and males, ranking eighth and 13th in the world, respectively. ${ }^{3}$ Although treatments for anxiety disorders are effective, ${ }^{4}$ not everyone with anxiety will receive appropriate treatment. ${ }^{5}$ Moreover, treatment alone is not sufficient to eliminate the disease burden imposed by anxiety disorders. ${ }^{6}$ It will be very difficult to decrease this burden unless the incidence of new cases is reduced, and this is only possible through primary prevention.

Psychological and/or educational interventions are effective at preventing anxiety disorders. ${ }^{7}$ Most preventive programmes delivering cognitive behaviour therapy have been carried out in an academic

P Moreno-Peral, PhD, senior researcher:

S Conejo-Cerón, PhD, postdoc researcher, Primary

Care District of Málaga-Guadalhorce, Instituto de Investigación Biomédica de Málaga-IBIMA

Primary Care Prevention and Health Promotion Network (redlAPP), Spain. JD Luna, MD, PhD professor, Department of Biostatistics, Medical School, University of Granada, Granada, Spain. M King, MD, PhD, professor, Mental Health Sciences, Faculty of Brain Sciences; I Nazareth, MD, PhD, professor, Department of Primary Care and Population Health, UCL, London, UK. C Martín-Pérez, MD, PhD, medical doctor

Marquesado Health Centre, Northeast Area of Granada, Spain. C Fernández-Alonso, MD, PhD, medical doctor, Service Assistance Programs, Regional Health Management Valladolid, Spain. MI Ballesta-Rodríguez, MD, PhD, family doctor Federico del Castillo Health Centre, Jaén, Spain A Fernández, $\mathrm{PhD}$, senior researcher, Parc Sanitari Sant Joan de Déu, Fundació Sant Joan de Déu, Barcelona, Spain; CIBERESP, Centro de Investigacion Biomedica en Red de Epidemiologia y Salud Publica, Spain. JM Aiarzaguena, MD, PhD, family doctor, San Ignacio Health Centre, Primary Care Research Unit, Osakidetza Bilbao, setting and administered by psychologists. Four trials on anxiety prevention have been conducted in primary care, and only two implemented by GPs. ${ }^{7}$ The primary healthcare setting is ideal for preventing the onset of illnesses, such as anxiety disorders, because it is easily accessible, provides continuity of care, and is used by a large proportion of the population. ${ }^{8,9}$ To the authors' knowledge, there are no interventions administered by GPs to prevent the onset of anxiety disorders in the adult population irrespective of people's individual risk levels (universal prevention).7.10

Anxiety and depression frequently occur together, ${ }^{11}$ share most of the same risk factors, ${ }^{12-14}$ and respond to the same treatments ${ }^{15}$ and preventive interventions. ${ }^{16,17}$ The authors' research group (the predictD group) developed a personalised novel biopsychosocial intervention, the predictDintervention, based on the patient's individual level of risk and risk profile of depression, which can be implemented by GPs to

Spain. C Montón-Franco, MD, PhD, family doctor Casablanca Health Centre, Aragonese Institute of Health Sciences, IIS Aragón, Department of Medicine and Psychiatry, University of Zaragoza, Spain. JA Bellón, MD, PhD, family doctor, El Palo Health Centre, Andalusian Health Service (SAS); Primary Care District of Málaga-Guadalhorce; Biomedical Research Institute of Málaga (IBIMA); Primary Care Prevention and Health Promotion Network (redIAPP); Department of Public Health and Psychiatry, University of Málaga, Spain.

\section{Address for correspondence}

Patricia Moreno-Peral, Primary Care District of Málaga-Guadalhorce, Instituto de Investigación Biomédica de Málaga-IBIMA, C/ Sevilla, 23, 29009 Málaga, Spain.

Email: predictmalagadhotmail.com

Submitted: 27 March 2020; Editor's response: 22 May 2020; final acceptance: 12 August 2020 (c)The Authors

This is the full-length article (published online 26 Jan 2021) of an abridged version published in print. Cite this version as: $\mathbf{B r} \mathbf{J}$ Gen Pract 2021; DOI: https://doi.org/10.3399/bjgp20X714041 


\section{How this fits in}

To date, very few studies on the prevention of anxiety have been conducted in primary care and only two of these interventions were performed by GPs. In the predictDintervention, the GP informed each patient about their level of risk (probability) and specific risk factors for depression, and they agreed on a personalised biopsychosocial intervention to prevent depression Iconstituting different strategies for dealing with the risk factors of each patient and encouraging them to have healthy lifestyle habits and promote their personal resources). The predictD-intervention reduced the occurrence of new cases of major depression compared with usual GP care, although this reduction was modest. This secondary study showed that the predictD-intervention also had a modest effect in preventing anxiety at 18 months. The predictD-intervention seems promising, although further studies are needed to confirm and even improve these results.

prevent the onset of major depression. ${ }^{18}$ This intervention provided a modest reduction in the incidence of major depression compared with usual care. ${ }^{19}$ It was not statistically significant, but it had a relevant cost utility. ${ }^{20}$ The aim of this secondary study was to assess whether the predictD-intervention for the prevention of depression was effective at preventing anxiety in patients in primary care who did not have depression or anxiety.

\section{METHOD}

\section{Design and setting}

The predictD-Cluster, Controlled, Randomised Trial (predictD-CCRT) ${ }^{18}$ had two parallel groups (the predictD-intervention and usual care), with cluster assignment by practice, and 18-month follow-up. It was conducted in seven Spanish cities (Malaga Granada, Jaen, Saragossa, Salamanca, Bilbao, and Barcelonal between October 2010 and July 2012.

The Spanish National Health Service provides universal health coverage for citizens through a public system financed by taxes and is free at the point of use. Each patient is assigned to only one GP, who functions as a gatekeeper to the wider system. Patients can visit their GP as often as they want without having to pay for consultations, even when they do so for preventive reasons. Details of the trial design are provided elsewhere. ${ }^{18-20}$

\section{Participants}

Ten practices in each city and two GPs in each practice were randomly selected using closed opaque envelopes by an independent investigator who was centrally located but not part of the research team.

Four to six patients per day were randomly selected from among the patients who had recently been seen at the practices by independent research assistants using random numbers. GPs reviewed the lists of patients to identify those who met the exclusion criteria. This process continued until there were 26 to 27 eligible patients for each GP. The recruitment was performed from October 2010 to February 2011. All eligible patients were invited to participate, and those who agreed to do so were informed about the study by research assistants. Exclusion criteria for patients were age $<18$ or $>75$ years; inability to understand or speak Spanish; documented severe mental disorder (such as psychosis, bipolar disorder, or personality disorder), cognitive impairment, or terminal illness; being scheduled to be out of the city more than 4 months during the 18 months of follow-up; and representatives attending the surgery on behalf of the patient. Trained and independent interviewers administered the Composite International Diagnostic Interview (Depression section) ${ }^{21,22}$ at baseline, and patients with a diagnosis of major depression during the previous 6 months were also excluded from the trial. For this secondary study, patients were removed if they had an anxiety syndrome in the previous 6 months, according to the Primary Care Evaluation of Mental Disorders (PRIME-MD-anxiety) questionnaire. ${ }^{23,24}$

\section{Randomisation and blinding}

In each city, five practices were assigned to the control group and five to the intervention group. This random allocation was achieved using closed opaque envelopes by an independent investigator who was centrally located but not part of the research team. GPs and patients were not blind to group allocations. The interviewers who assessed outcomes and investigators who did the statistical analyses were blinded to group allocations.

\section{Intervention}

The predictD-intervention has been described in detail elsewhere. ${ }^{18,19}$ Before delivering the intervention, GPs attended a 10- to 15-hour training workshop (see Supplementary Annex S1 for details). GPs communicated to each patient their risk factors for depression and overall probability of developing depression using the Spanish version of the predictD algorithm ${ }^{12,25}$ (http:// www.predictplusprevent.com/Calculadora. 
php?idioma=en). They then gave patients a patient-oriented booklet about preventing depression; enabling patients to actively deal with their risk factors, and feel empowered; and constructed a tailored biopsychosocial intervention for each patient to prevent depression (see Supplementary Annex S2 for details about the different components of the predictD intervention, and Supplementary Figure S1 for a theoretical model for the prevention of depression in primary care). GPs were given recommendations for conducting a GP-patient interview to prevent depression (see Supplementary Box S1 for details) and were directed to a 5-minute video showing a GP delivering an intervention online (see Supplementary Figure S2 for an example of the mechanisms provided by the GP to reduce the likelihood of becoming depressed after the intervention). GPs delivered interventions to patients at baseline and at 6- and 12-month follow-up visits, which each lasted 10-15 minutes. Patients in the predictD-intervention group also continued to receive usual care.

GPs in the control group did not receive information about their patients' profiles and levels of risk, nor did they attend the training workshop. Patients in the control group continued to receive usual care, and were assessed for depression, anxiety, and other information at the same intervals as patients in the intervention group.

\section{Outcomes}

The endpoint of the predictD-CCRT study was cumulative incidence of major depression at 18 months. The endpoint for this secondary study was the cumulative incidence of anxiety syndromes at 18 months measured at 6, 12, and 18 months using the PRIME-MD questionnaire. ${ }^{23}$ The Spanish version of PRIME-MD can classify patients who test positive for panic attacks, generalised anxiety, and other anxiety syndromes. ${ }^{24} \mathrm{~A}$ dichotomous anxiety variable was used to indicate when any of the three anxiety syndromes are present in a given patient. The cumulative incidence of anxiety syndromes was also evaluated at 6 and 12 months. It should be noted that the PRIME-MD instrument is not recorded on ClinicalTrials.gov as a secondary outcome; however, it can be found in the protocol publication of this study. ${ }^{18}$ Additional information collected from patients, GPs, and practices is described in detail elsewhere (see Supplementary Annex S3 for a summary). ${ }^{18-20}$ All patient variables were assessed at baseline and at 6, 12, and 18 months in both study groups. GPs participating in the trial completed a selfadministered questionnaire at baseline.

\section{Statistical analysis}

All analyses were performed using Stata (version 13.1) and participants were analysed according to their randomised group. The cumulative incidence of anxiety at 18 months in each study group was compared using generalised estimating equations to account for the cluster randomised design, with multiple imputation to account for missing outcomes. Generalised estimating equations were fitted with a binomial-family, logit-link function; terms for intervention group and baseline probability of depression were included; and an exchangeable correlation structure and robust standard errors were included for clustering on practice, whose intraclass correlation coefficient was 0.029 . The statistical power of the secondary study sample was calculated a posteriori, and was $33.2 \%$ lrho $=0.029$; alpha $=0.05$; incidence difference $=-0.0267, \mathrm{~N} 1=1514, \mathrm{~N} 2=1484$, number of clusters $\mathrm{K} 1=35$ and $\mathrm{K} 2=35$, average number of patients per cluster $M 1=43.26$ and $M 2=42.4$ ). It was decided a priori to adjust for baseline probability of depression ${ }^{18}$ because it was considered strongly predictive of the outcome and thus clinically prognostic. 26,27 Standardised probabilities of anxiety during the 18-month study were calculated using the margins in Stata. Missing outcomes were accounted for using multiple imputations with chained equations, ${ }^{28}$ under a missing-at-random framework. Fifty imputed samples were generated and estimates were combined using Rubin rules. ${ }^{29}$

Sensitivity analysis included the unadjusted incidence of anxiety at 18 months; and the incidence of anxiety at 18 months adjusted for all unbalanced variables. Supplementary analyses were also carried out to evaluate the cumulative incidence of anxiety at 6 and 12 months.

All $P$-values were two-sided and considered significant at $\leq 0.05$. All confidence intervals (CI) were reported at $95 \%$.

\section{RESULTS}

Eligible patients at each stage of the study up to 18 months are shown in Figure 1 (see Supplementary Figure S3 for selection of primary care centres and GPs). A total of $68.7 \%(n=1889)$ patients agreed to participate in the control group, and $76.1 \%(n=1894)$ agreed to participate in the intervention group. Of the 1453 patients who declined to participate, $72.1 \%(n=1048)$, 


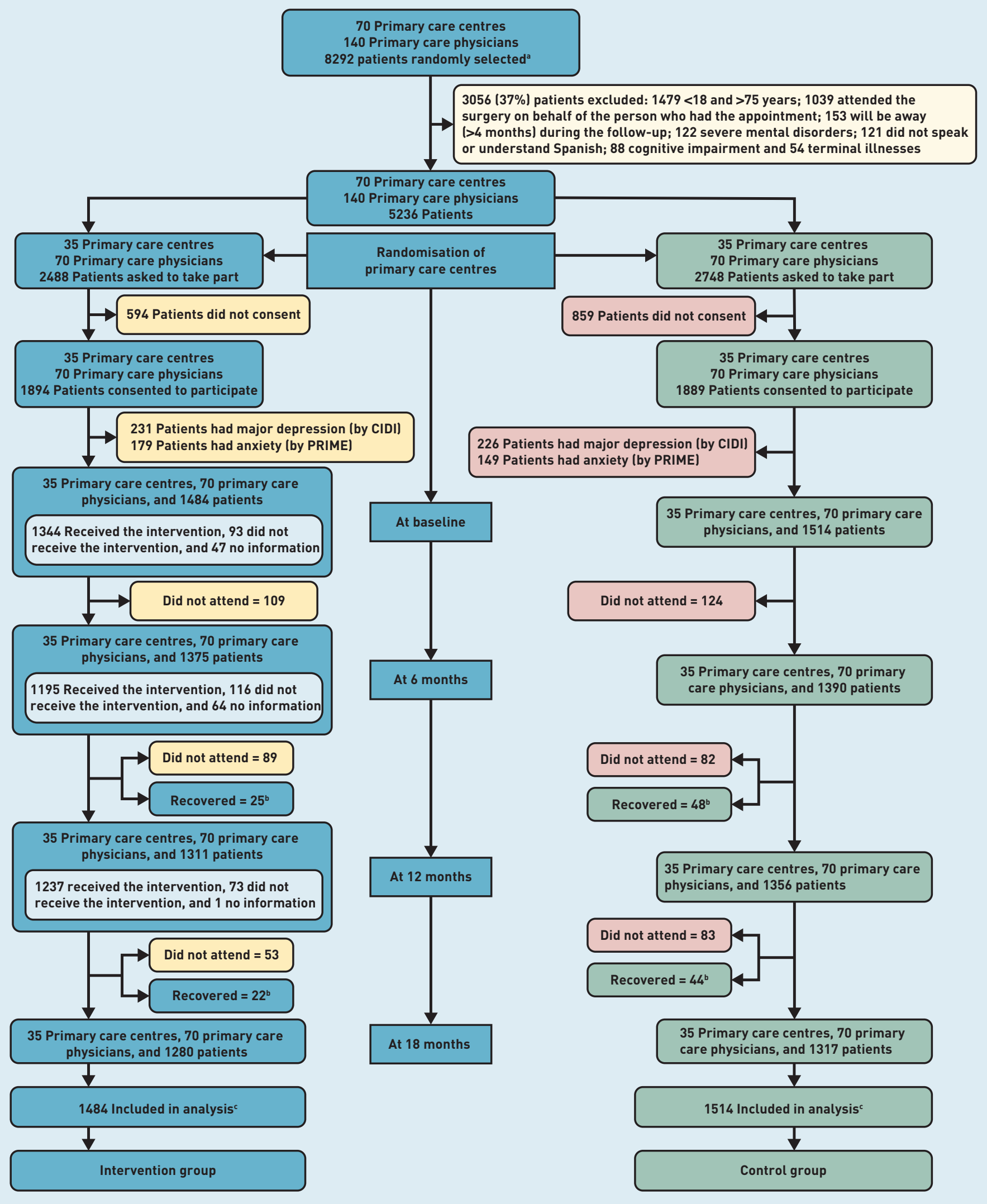

Figure 1. Study design and operation.

a Systematic random sampling, from the primary care physicians' appointment lists at random starting points for each day and without replacement. ${ }^{\text {} P a t i e n t s ~ w h o ~ a t t e n d e d ~}$ the respective evaluation point, but did not attend any previous point. 'Analyses conducted using multiple imputation to account for missing outcomes (240 intervention and 250 control patients had missing outcome at 18 months). CIDI = Composite International Diagnostic Interview. 


\section{Table 1. Baseline characteristics of the primary care centres included in the study}

\begin{tabular}{lcc} 
Variable & $\begin{array}{c}\text { Control group } \\
(\boldsymbol{n}=\mathbf{3 5})^{\mathbf{a}}\end{array}$ & $\begin{array}{c}\text { Intervention } \\
\text { group }(\boldsymbol{n}=\mathbf{3 5})^{\mathbf{a}}\end{array}$ \\
\hline Years in operation, mean (SD) & $18.9(9.90)$ & $20.5(7.29)$ \\
\hline Enrolled population, mean (SD) & $19992(6739)$ & $20331(10014)$ \\
\hline Number of primary care physicians, mean (SD) & $11.6(3.94)$ & $12.1(5.83)$ \\
\hline Number of primary care paediatricians, mean (SD) & $2.5(1.04)$ & $2.6(1.31)$ \\
\hline Number of primary care nurses, mean (SD) & $12.0(4.08)$ & $12.3(5.33)$ \\
\hline Primary care social workers, $\boldsymbol{n}(\%)$ & $19(54.3)$ & $19(45.7)$ \\
Half-time or less & $16(45.7)$ & $19.3)$ \\
\hline
\end{tabular}

${ }^{a}$ There were no missing values. $S D=$ standard deviation anxiety. In the intervention group, 1244 (83.8\%) participants were evaluated for cumulative incidence of anxiety and 240 (16.2\%) participants had missing outcomes in the cumulative incidence of anxiety land were imputed later). In the control group, 1264 (83.5\%) participants were evaluated for cumulative incidence of anxiety and 250 (16.5\%) participants had missing outcomes in the cumulative incidence of anxiety land were also imputed later).

The predictD-intervention was effective at preventing anxiety at 18 months, because $10.4 \%$ of patients in the intervention group $(95 \% \mathrm{Cl}=8.7 \%$ to $12.1 \%)$ developed anxiety compared with $13.1 \%$ in the control group (95\% Cl $=11.4 \%$ to $14.8 \%$ ) labsolute difference $=-2.67$ percentage points; $95 \%$ $\mathrm{Cl}=-5.05$ to -0.28 percentage points; $P=0.029$ ) (Table 3). The intervention was not statistically significant for prevention of anxiety at 6 months or 12 months, although the effectiveness seemed to increase over time (Table 3). The unadjusted analysis was not statistically significant, whereas the analysis adjusted for baseline depression plus additional covariates slightly increased the effectiveness and was statistically significant labsolute difference $=-2.78$ percentage points; $95 \% \mathrm{Cl}=-4.95$ to -0.62 percentage points; $P=0.012$, Table 3).

GPs reported no adverse effects associated with the intervention. Three patients in the intervention group contacted researchers with complaints about their GPs and to request a change of GP.

\section{DISCUSSION}

\section{Summary}

A personalised and novel intervention based on the level of risk and risk profile of depression involving adult patients at low, moderate, and high risk, and implemented by GPs, was effective in reducing the incidence of anxiety syndromes at 18 months. The results of sensitivity analyses were consistent with a modest but robust effect.

\section{Strengths and limitations}

This is the first randomised trial to evaluate the effectiveness of an intervention administered by GPs to prevent depression, which was effective in reducing the onset of anxiety disorders in the adult population. This novel intervention allows for both disorders to be addressed. A large sample of patients were recruited irrespective of their individual risk levels (universal prevention). Furthermore, the trial was delivered by GPs in their practices and was based on usual components of primary care; therefore, it 
Table 2. Baseline characteristics of the family physicians involved in the study

\begin{tabular}{|c|c|c|}
\hline Variable & $\begin{array}{c}\text { Control group }(N=70) \\
n(\%) \text { or mean }(S D)\end{array}$ & $\begin{array}{c}\text { Intervention group }(N=70) \\
n(\%) \text { or mean (SD) }\end{array}$ \\
\hline Sex (male) & $29(41.4 \%)^{b}$ & $28(41.2 \%)^{d}$ \\
\hline Age lyears) & $53.8(5.97)^{d}$ & $52.1(7.13)^{\mathrm{e}}$ \\
\hline Size of town where practice located & d & $d$ \\
\hline 2500-30 000 inhabitants & $10(14.7 \%)$ & $13(19.1 \%)$ \\
\hline $30001-200000$ inhabitants & $10(14.7 \%)$ & $11(16.2 \%)$ \\
\hline$>200000$ inhabitants & $48(70.6 \%)$ & $44(64.7 \%)$ \\
\hline Primary care physician list size & $1581(166.02)^{\mathrm{f}}$ & $1538(244.95)^{9}$ \\
\hline Year doctor qualified & $1983(5.84)^{b}$ & 1984 (7.07)e \\
\hline Time working at the health centre (months) & $87.1(46.20)^{b}$ & $79.8(45.3)^{d}$ \\
\hline Average time spent per patient $<10$ minutes $^{a}$ & $39(55.7 \%)^{b}$ & $41(60.3 \%)^{d}$ \\
\hline Relationship with mental health team (good/very good) & $48(70.6 \%)^{d}$ & $36(53.7 \%)^{e}$ \\
\hline Satisfaction with support from mental health team & c & d \\
\hline Very dissatisfied/dissatisfied & $15(21.7 \%)$ & $14(20.6 \%)$ \\
\hline Neither dissatisfied nor satisfied & $19(27.5 \%)$ & $25(36.8 \%)$ \\
\hline Satisfied/very satisfied & $35(50.7 \%)$ & $29(42.6 \%)$ \\
\hline Ease and familiarity with handling antidepressants & b & d \\
\hline Very uncomfortable/uncomfortable/neither uncomfortable nor comfortable & $10(14.3 \%)$ & $18(26.5 \%)$ \\
\hline Comfortable/very comfortable & $60(85.7 \%)$ & $50(73.5 \%)$ \\
\hline Relationship with primary care nurses (good/very good) & $62(88.6 \%)^{b}$ & $59(86.8 \%)^{d}$ \\
\hline Active role of primary care nurse with patients with mental health disorders & b & d \\
\hline Strongly disagree/disagree & $22(31.4 \%)$ & $30(44.1 \%)$ \\
\hline Neither disagree nor agree & $23(32.9 \%)$ & $20(29.4 \%)$ \\
\hline Agree/strongly agree & $25(35.7 \%)$ & $18(26.5 \%)$ \\
\hline Relationship with primary care social workers (good/very good) & $49(70 \%)^{b}$ & $45(66.2 \%)^{d}$ \\
\hline Active role of primary care social worker with patients with mental health disorders & b & d \\
\hline My team has no primary care social worker & $6(8.6 \%)$ & $8(11.4 \%)$ \\
\hline Strongly disagree/disagree & $16(22.9 \%)$ & $14(20.0 \%)$ \\
\hline Neither disagree nor agree & $23(32.9 \%)$ & $27(38.6 \%)$ \\
\hline Agree/strongly agree & $24(34.3 \%)$ & $18(25.7 \%)$ \\
\hline \multicolumn{3}{|l|}{ Style of professional practice } \\
\hline Job satisfaction (range 4-20) & $16.44(2.32)^{b}$ & $16.09(2.32)^{d}$ \\
\hline Perception workload (range 4-20) & $14.67(2.74)^{\mathrm{e}}$ & $14.26(3.65)^{d}$ \\
\hline Biomedical versus psychosocial orientation (range 4-20) & $10.25(2.96)^{d}$ & $10.38(3.56)^{d}$ \\
\hline Extraversion (EPQR-A) Low (0 to 4 score) & $35(51.5 \%)^{d}$ & $38(57.6 \%)^{\dagger}$ \\
\hline Neuroticism (EPQR-A) Low (0 to 4 score) & $67(95.7 \%)^{b}$ & $64(95.5 \%)^{\mathrm{e}}$ \\
\hline Psychoticism (EPQR-A) Low (0 to 4 score) & $68(98.6 \%)^{c}$ & $63(94.0 \%)^{\mathrm{e}}$ \\
\hline Long-term contract (yes) & $65(92.9 \%)^{b}$ & $63(92.6 \%)^{d}$ \\
\hline Accredited to train residents (yes) & $37(52.9 \%)^{\mathrm{b}}$ & $37(54.4 \%)^{d}$ \\
\hline Training fourth-year resident (yes) & $25(35.7 \%)^{b}$ & $24(35.3 \%)^{d}$ \\
\hline Training first-year resident (yes) & $17(24.6 \%)^{c}$ & $16(22.9 \%)^{\mathrm{e}}$ \\
\hline Three-to-four year postgraduate training (yes) & $41(58.6 \%)^{b}$ & $42(61.8 \%)^{d}$ \\
\hline Member of the Communication \& Health group (yes) & $6(8.6 \%)^{b}$ & $5(7.4 \%)^{d}$ \\
\hline
\end{tabular}

a Regardless of time per patient assigned on the agenda, what is your average time spent per patient $<10$ minutes or $\geq 10$ minutes)? Missing values, number (\%): ${ }^{b}(0 \%)$; 1 (1.4\%);

${ }^{d} 2(2.8 \%) ; \bullet 4(4.3 \%) ; 4(5.7 \%) ; 95(7.1 \%)$. SD $=$ standard deviation.

will require little adaptation. This study had several limitations that must be taken into account. The predictD-intervention was performed with the aim of preventing major depression; therefore, this study addresses a secondary objective. The questionnaire used to evaluate the outcome, PRIME-MD, has good reliability and validity indices, ${ }^{23-24}$ but it is not possible to rule out classification bias. Moreover, only a syndromic approach was considered as defined by the PRIMEMD Igeneralised anxiety, panic disorders, and non-specific anxiety).

Although patients were randomly selected by independent research assistants, a potential self-selection bias by patients was possible because there were more refusals to participate in the control group than in 


\section{Table 3. Effectiveness of the study intervention: proportion of patients with anxiety during the study ${ }^{\mathrm{a}}$}

\begin{tabular}{|c|c|c|c|c|}
\hline Variable & $\begin{array}{c}\text { Intervention group } \\
(n=1484, \%) \\
(95 \% \mathrm{Cl})\end{array}$ & $\begin{array}{l}\text { Control group } \\
(n=1514, \%) \\
(95 \% \mathrm{Cl})\end{array}$ & $\begin{array}{l}\text { Absolute difference } \\
\% \text { points }(95 \% \mathrm{Cl})\end{array}$ & $P$-value \\
\hline \multicolumn{5}{|l|}{ Primary analysis } \\
\hline Anxiety at 18 months & 10.43 (8.73 to 12.13 ) & 13.10 (11.4 to 14.79$)$ & $-2.67(-5.05$ to -0.28$)$ & 0.029 \\
\hline \multicolumn{5}{|l|}{ Secondary analysis } \\
\hline Anxiety at 6 months & 4.55 (3.16 to 5.95 ) & 5.18 (4.04 to 6.32$)$ & $-0.63(-2.43$ to 1.17$)$ & 0.492 \\
\hline Anxiety at 12 months & 7.99 (6.43 to 9.55) & 9.56 (8.14 to 10.98 ) & $-1.57(-3.67$ to 0.54$)$ & 0.145 \\
\hline \multicolumn{5}{|l|}{ Sensitivity analysis } \\
\hline $\begin{array}{l}\text { Anxiety at } 18 \text { months, } \\
\text { unadjusted }\end{array}$ & 10.69 (8.91 to 12.46$)$ & 12.83 (10.88 to 14.79$)$ & $-2.14(-4.78$ to 0.50$)$ & 0.112 \\
\hline $\begin{array}{l}\text { Anxiety at } 18 \text { months, } \\
\text { adjusted for all } \\
\text { unbalanced variables }^{\mathrm{b}}\end{array}$ & 10.36 (8.81 to 11.91 ) & 13.14 (11.62 to 14.67$)$ & $-2.78(-4.95$ to -0.62$)$ & 0.012 \\
\hline
\end{tabular}

aisplays standardised probabilities or predicted margins estimated using generalised estimating equations including an exchangeable correlation structure and robust standard errors for clustering on centre and adjusted for baseline probability of depression. ${ }^{b}$ Adjusted for baseline probability of depression, the other unbalanced baseline variables not included in the predictD-Spain risk algorithm lemployment status, ownerloccupier of an accommodation, perception of safety inside/outside the home, and experiences of discrimination), GPs' familiarity and ease in their relationships with mental health teams, social workers, nurses, and use of antidepressants, and city. $\mathrm{Cl}=$ confidence interval.

\section{Funding}

This study was supported by the Spanish Ministry of Health, the Institute of Health Carlos III, and the European Regional Development Fund 'Una manera de hacer Europa' Igrant FIS reference: PI12/02755, PS09/02272, PS09/02147, PS09/01095, PS09/00849, and PS09/00461), the Andalusian Council of Health Igrant reference: 0583/2012, PI-0569-2010), and the Prevention and Health Promotion Research Network 'redIAPP' (RD16/0007).

\section{Ethical approval}

The predictD-CCRT Study complies with the Declaration of Helsinki. This trial was approved by the following ethics committees in each participating city: Ethics Committee on Human Research of the University of Granada, Ethics and Research Committee of Primary Health District of Málaga, Ethics Committee on Clinical Research of Sant Joan de Déu Foundation (Barcelona) (PIC CEIC-6209), Ethics Committee of Clinical Research of Aragon (CP06/05/2009), Ethics Committee for Health Research of the Jaén Hospital, Ethics Committee for Clinical Research of Euskadi (03/2009), and Ethics Committee for Clinical Research of the Rio Hortega Hospital of Valladolid (04/2009). Trial registration: ClinicalTrials.gov identifier: NCT01151982. the intervention group. This, along with the cluster randomisation, could explain the relative imbalance at the patient level.30,31 Patients in the control group were more satisfied with home life and felt safer than patients in the intervention group, which might have made them generally less likely to develop an anxiety disorder. Similarly, control GPs were more comfortable in their relationships with mental health teams, social workers, nurses, and use of antidepressants than were intervention GPs. When all these variables were adjusted for in the sensitivity analysis, the effectiveness in preventing anxiety was slightly increased (Table 3). Finally, the sample possibly underrepresented patients who are treated infrequently; ${ }^{32}$ however, those who are seen frequently are more likely to develop anxiety disorders, ${ }^{33}$ and therefore have the most need for preventive strategies.

\section{Comparison with existing literature}

An intervention to prevent major depression that also reduces the incidence of anxiety syndromes could be explained by the fact that depression and anxiety share most of the same risk factors, ${ }^{12,13}$ and it is also possible that both are expressions of a latent pathological process. ${ }^{34}$ Many interventions have been developed for the prevention of both anxiety and depression disorders, showing successful results in both cases. ${ }^{15,16}$ Transdiagnostic interventions seem to be a promising approach, and those aimed at preventing both depression and anxiety are increasing. ${ }^{35,36}$ The clinical practice guides consider the use of antidepressants as one of the treatments of choice for generalised anxiety disorders and panic disorder. ${ }^{37,38}$

It is possible that the preventive effect on depression of the predictD-intervention generated the reduction in anxiety through the modification of a set of shared risk between anxiety and depression. ${ }^{39}$ Moreover, the mediators of psychological and psychoeducational interventions for the prevention of depression and anxiety are quite similar, being the change in cognitions as the main mediator of both conditions. ${ }^{40}$ Another non-exclusive hypothesis might be that the predictD-intervention first reduced the incidence of anxiety and then the incidence of depression or vice versa. Evidence indicates that anxiety disorders temporally precede depression in most comorbid cases $^{41}$ and that treatment for an anxiety disorder also produces declines in mood disorders. ${ }^{42}$ On the other hand, it might be that the predictD-intervention improves people's mental health in general, leading to prevention of anxiety and depression at once.

The reduction in anxiety syndromes was about the same size as reductions reported in other studies that have evaluated psychological interventions to reduce separately the incidence of anxiety and depression. ${ }^{7,43,44}$ However, several studies found greater reductions in the incidence of panic disorder. .5. $^{4} 6$

The reduction of the incidence of anxiety in the current study seemed to increase over time, which might be due to a doseresponse effect of the intervention or simply a need for time and the accumulation of intervention visits to create the changes needed to prevent anxiety. A similar finding was observed for the reduction of the incidence of depression through the predictD-intervention, ${ }^{19}$ but this was not so in other interventions for the primary prevention of anxiety. ${ }^{7,47,48}$

Most studies into the prevention of anxiety and depression examined interventions with a cognitive behaviour orientation, which have been administered by psychologists. ${ }^{7,43,44}$ In the current study, the predictD-intervention is based on each patient's individual risk for major depression, identifies specific risk factors for depression in each patient that are amenable to change, and helps the patient use this information to improve knowledge and alter behaviour. Furthermore, it is delivered by GPs in their practices. 


\section{Provenance}

Freely submitted; externally peer reviewed.

\section{Competing interests}

The authors have declared no competing interests.

\section{Acknowledgements}

Thanks to the Primary Care District of Malaga, the Institute of Health Carlos III (ISCIII), European Regional Development Fund (ERDF), the Andalusian Public Foundation for Health and Biomedicine Research in Malaga (FIMABIS), and the Spanish Network of Primary Care Research (redlAPP) for their support. This work would not have been possible without the participation of the patients and family physicians involved in the trial.

\section{Data sharing}

Individual participant data that underlie the results reported in this article will be made available on request after de-identification. The proposed use of the data must be approved by an independent review committee.

\section{Open access}

This article is Open Access: CC BY-NC 4.0 licence (http://creativecommons.org/ licences/by-nc/4.0/).

\section{Discuss this article}

Contribute and read comments about this article: bjgp.org/letters

\section{Implications for research and practice}

A personalised intervention based on the level and risk profile of depression implemented by GPs provided a modest but statistically significant reduction in the incidence of anxiety. Although the effect size was a small decrease in anxiety incidence in the predictD-intervention group in comparison with the usual-care group), these relative numbers could be clinically relevant in absolute terms. From the perspective of public health, small effects on prevention could have a high impact, avoiding anxiety and depression, improving quality of life, and reducing costs, if the interventions are cost-effective and scalable to a large number of people, which is possible in a primary care setting. From this perspective, and bearing in mind the findings, healthcare systems could be encouraged to implement and disseminate prevention programmes for both anxiety and depression disorders rather than for each disorder alone. ${ }^{49}$ The fact that the predictD-intervention was cost-effective for the prevention of depression when delivered by GPs in their practices ${ }^{20}$ would facilitate its implementation. However, it remains to be clarified whether universal prevention of anxiety and depression in primary care is more cost-effective and acceptable for GPs and patients than selective prevention in patients who are high-risk.
In general, patients were pleased to be informed about their risk for depression, ${ }^{50}$ and the GPs had a positive experience with the predictD-intervention, as it was easily embedded into their practice. ${ }^{51}$ They perceived it useful as a biopsychosocial approach for improving the emotional health of patients and their relationship with them, as well as their own satisfaction as a GP. However, they also detected some barriers such as lack of time, and the need for specific training to effectively communicate the risk of developing depression..$^{51}$ For the future implementation of the predictDintervention, GPs suggested intervention based on level of risk. From their point of view, having to carry out an intervention in all patients regardless of their level of risk (universal prevention) is an unrealistic workload. ${ }^{51}$

It is not known how important patient initiative (dealing with their risk factors, overcoming difficulties, as well as starting healthy behaviours) and empowerment were in the predictD-intervention, nor which or how many of the components of the intervention were involved in each case. Studies to define the active ingredients of the intervention are therefore also necessary. The predictD-intervention seems promising, but further studies to confirm and even improve these results are needed. 


\section{REFERENCES}

1. Steel Z, Marnane C, Iranpour C et al. The global prevalence of common mental disorders: a systematic review and meta-analysis 1980-2013. Int J Epidemiol 2014; 43(2): 476-493

2. Serrano-Blanco A, Palao DJ, Luciano JV, et al. Prevalence of mental disorders in primary care: results from the diagnosis and treatment of mental disorders in primary care study (DASMAP). Soc Psychiatry Psychiatr Epidemiol 2010; 45(2): 201-210.

3. GBD 2017 Disease and Injury Incidence and Prevalence Collaborators. Global, regional, and national incidence, prevalence, and years lived with disability for 354 diseases and injuries for 195 countries and territories, 1990-2017: a systematic analysis for the Global Burden of Disease Study 2017. Lancet 2018; 392(10159): 1789-1858.

4. Barlow DH, Lehman CL. Advances in the psychosocial treatment of anxiety disorders. Implications for national health care. Arch Gen Psychiatry 1996; 53(8): 727-735.

5. Fernández A, Haro JM, Martinez-Alonso M, et al. Treatment adequacy for anxiety and depressive disorders in six European countries. Br J Psychiatry 2007; 190: 172-173.

6. Andrews G, Issakidis C, Sanderson K, et al. Utilising survey data to inform public policy: comparison of the cost-effectiveness of treatment of ten mental disorders. Br J Psychiatry 2004; 184: 526-533.

7. Moreno-Peral P, Conejo-Cerón S, Rubio-Valera M, et al. Effectiveness of psychological and/or educational interventions in the prevention of anxiety: a systematic review, meta-analysis, and meta-regression. JAMA Psychiatry 2017; 74(10): 1021-1029.

8. Starfield B, Shi L, Macinko J. Contribution of primary care to health systems and health. Milbank Q2005; 83(3): 457-502.

9. World Health Organization. The world health report 2008 - primary health care (now more than ever). Geneva: WHO, 2008. https://www.who.int/whr/2008/en/ (accessed 2 Dec 2020).

10. García-Campayo J, Lopez del Hoyo Y, Salas-Valero M, et al. Primary prevention of anxiety disorders in primary care: a systematic review. Prev Med 2015; 76 Suppl: S12-S15

11. Kessler RC, Angermeyer M, Anthony JC, et al. Lifetime prevalence and ageof-onset distributions of mental disorders in the World Health Organization's World Mental Health Survey Initiative. World Psychiatry 2017; 6(3): 168-176.

12. Bellón JA, de Dios Luna J, King M, et al. Predicting the onset of major depression in primary care: international validation of a risk prediction algorithm from Spain. Psychol Med 2011; 41(10): 2075-2088.

13. Moreno-Peral P, de Dios Luna J, Marston L, et al. Predicting the onset of anxiety syndromes at 12 months in primary care attendees. The predictA-Spain study. PLoS One 2014; 9(9): e106370.

14. Moreno-Peral $\mathrm{P}$, Conejo-Cerón S, Motrico E, et al. Risk factors for the onset of panic and generalised anxiety disorders in the general adult population: a systematic review of cohort studies. J Affect Disord 2014; 168: 337-348.

15. Axelson DA, Birmaher B. Relation between anxiety and depressive disorders in childhood and adolescence. Depress Anxiety 2001; 14(2): 67-78.

16. Stockings EA, Degenhardt L, Dobbins T, et al. Preventing depression and anxiety in young people: a review of the joint efficacy of universal, selective and indicated prevention. Psychol Med 2016; 46(1): 11-26

17. van't Veer-Tazelaar PJ, van Marwijk HWJ, van Oppen P, et al. Stepped-care prevention of anxiety and depression in late life: a randomized controlled trial. Arch Gen Psychiatry 2009; 66(3): 297-304

18. Bellón JÁ, Conejo-Cerón S, Moreno-Peral P, et al. Preventing the onset of major depression based on the level and profile of risk of primary care attendees: protocol of a cluster randomised trial (the predictD-CCRT study). BMC Psychiatry 2013; 13: 171

19. Bellón JA, Conejo-Cerón S, Moreno-Peral P, et al. Intervention to prevent major depression in primary care: a cluster randomized trial. Ann Intern Med 2016; 164(10): $656-665$

20. Fernández A, Mendive JM, Conejo-Cerón S, et al. A personalized intervention to prevent depression in primary care: cost-effectiveness study nested into a clustered randomized trial. BMC Med 2018; 16(1): 28.

21. Robins LN, Wing J, Wittchen HU, et al. The Composite International Diagnostic Interview. An epidemiologic instrument suitable for use in conjunction with different diagnostic systems and in different cultures. Arch Gen Psychiatry 1988; 45(12): 1069-1077.
22. Wittchen HU. Reliability and validity studies of the WHO - Composite International Diagnostic Interview (CIDI): a critical review. J Psychiatr Res 1994: 28(1): 57-84.

23. Spitzer RL, Kroenke K, Williams JB. Validation and utility of a self-report version of PRIME-MD: the PHQ primary care study. Primary Care Evaluation of Mental Disorders. Patient Health Questionnaire. JAMA 1999; 282(18): 1737-1744.

24. Baca E, Saiz J, Agüera L, et al. Validation of the Spanish version of PRIMEMD: a procedure for diagnosing mental disorders in primary care. [Article in Spanish]. Actas Esp Psiquiatr 1999; 27(6): 375-383.

25. King M, Walker C, Levy G, et al. Development and validation of an international risk prediction algorithm for episodes of major depression in general practice attendees: the PredictD study. Arch Gen Psychiatry 2008; 65(12): 1368-1376.

26. Raab GM, Day S, Sales J. How to select covariates to include in the analysis of a clinical trial. Control Clin Trials 2000; 21(4): 330-342.

27. Wright N, Ivers N, Eldridge $S$, et al. A review of the use of covariates in cluster randomized trials uncovers marked discrepancies between guidance and practice. J Clin Epidemiol 2015; 68(6): 603-609.

28. Royston P. Multiple imputation of missing values: update of ice. Stata J 2005; 5(4): 527-536.

29. Rubin DB. Multiple imputation for nonresponse in surveys. New York, NY: Wiley, 2004

30. Hayes RJ, Moulton LH. Cluster randomized trials. New York, NY: CRC Press, 2009

31. Puffer S, Torgerson D, Watson J. Evidence for risk of bias in cluster randomised trials: review of recent trials published in three general medical journals. BMJ 2003; 327(7418): 785-789.

32. Lee ML, Yano EM, Wang M, et al. What patient population does visit-based sampling in primary care settings represent? Med Care 2002; 40(9): 761-770.

33. Wyke S, Hunt K, Walker J, Wilson P. Frequent attendance, socioeconomic status and burden of ill health. An investigation in the west of Scotland. Eur J Gen Pract 2003; 9(2): 48-55.

34. Mennin DS, Heimberg RG, Fresco DM, Ritter MR. Is generalized anxiety disorder an anxiety or mood disorder? Considering multiple factors as we ponder the fate of GAD. Depress Anxiety 2008; 25(4): 289-299.

35. Ruxandra Păsărelu C, Andersson G, Bergman Nordgren L, Dobrean A. Internet-delivered transdiagnostic and tailored cognitive behavioral therapy for anxiety and depression: a systematic review and meta-analysis of randomized controlled trials. Cogn Behav Ther 2017; 46(1): 1-28

36. Weisel KK, Zarski AC, Berger T, et al. Efficacy and cost-effectiveness of guided and unguided internet- and mobile-based indicated transdiagnostic prevention of depression and anxiety (ICare Prevent): a three-armed randomized controlled trial in four European countries. Internet Interv 2018; 16: 52-64.

37. National Institute for Health and Care Excellence. Anxiety: management of anxiety lpanic disorder, with or without agoraphobia, and generalised anxiety disorder) in adults in primary, secondary and community care. CG22. London: NICE, 2004

38. National Institute for Health and Care Excellence. Generalised anxiety disorder and panic disorder in adults: management. CG113. London: NICE, 2011. https:// www.nice.org.uk/guidance/cg113 (accessed 12 Jan 2021).

39. Titov N, Dear BF, Schwencke G, et al. Transdiagnostic internet treatment for anxiety and depression: a randomised controlled trial. Behav Res Ther 2011; 49(8): 441-452.

40. Moreno-Peral P, Bellon JA, Huibers MJH, et al. Mediators in psychological and psychoeducational interventions for the prevention of depression and anxiety. A systematic review. Clin Psychol Rev 2020; 76: 101813.

41. Wittchen HU, Beesdo K, Bittner A, Goodwin RD. Depressive episodes: evidence for a causal role of primary anxiety disorders? Eur Psychiatry 2003; 18(8): 384-393.

42. Tsao JCl, Mystkowski JL, Zucker BG, Craske MG. Impact of cognitive-behavioral therapy for panic disorder on comorbidity: a controlled investigation. Behav Res Ther 2005; 43(7): 959-970.

43. van Zoonen K, Buntrock C, Ebert DD, et al. Preventing the onset of major depressive disorder: a meta-analytic review of psychological interventions. Int J Epidemiol 2014; 43(2): 318-329.

44. Bellón JA, Moreno-Peral P, Motrico E, et al. Effectiveness of psychological and/ or educational interventions to prevent the onset of episodes of depression: a systematic review of systematic reviews and meta-analyses. Prev Med 2015; 76 Suppl: S22-S32. 
45. Gardenswartz CA, Craske MG. Prevention of panic disorder. Behav Ther 2001; 32(4): $725-737$.

46. Livermore N, Sharpe L, McKenzie D. Prevention of panic attacks and panic disorder in COPD. Eur Respir J 2010; 35(3): 557-563.

47. Zalta AK. A meta-analysis of anxiety symptom prevention with cognitivebehavioral interventions. J Anxiety Disord 2011; 25(5): 749-760.

48. Teubert D, Pinquart M. A meta-analytic review on the prevention of symptoms of anxiety in children and adolescents. J Anxiety Disord 2011; 25(8): 1046-1059
49. Beekman AT, de Beurs E, van Balkom AJ, et al. Anxiety and depression in later life: co-occurrence and communality of risk factors. Am J Psychiatry 2000; 157(1): 89-95.

50. Bellón JÁ, Moreno-Peral P, Moreno-Küstner B, et al. Patients' opinions about knowing their risk for depression and what to do about it. The predictDqualitative study. PLoS One 2014; 9(3): e92008.

51. Moreno-Peral P, Conejo-Cerón S, Fernández A, et al. Family physicians' views on participating in prevention of major depression. The predictD-EVAL qualitative study. PLoS One 2019; 14(5): e0217621. 